\title{
The Electronic Adiabatic-Diabatic Transformation Matrix: A Theoretical and Numerical Study of a Three-State System
}

\author{
Alexander Alijah \\ Departamento de Quìmica, Universidade de Coimbra, P-3049 Coimbra Codex, Portugal and Fakultät für \\ Chemie, Universität Bielefeld, Bielefeld, 33501 Germany \\ Michael Baer*,* \\ Institute of Atomic and Molecular Science, Taipei, Taiwan ROC 10764, and Department of Physics and \\ Applied Mathematics, Soreq NRC, Yavne 81800, Israel
}

Received: August 3, 1999; In Final Form: October 21, 1999

\begin{abstract}
In this work, we consider a diabatic $3 \times 3$ potential matrix which is used to study the three adiabaticdiabatic transformation angles that form the corresponding $3 \times 3$ adiabatic-diabatic transformation matrix. The three angles are known to be solutions of three coupled first-order differential equations (Top, Z. H.; Baer, M. J. Chem. Phys. 1977, 66, 1363). These equations are solved here for the first time and are shown to be stable and to yield meaningful solutions. Since many sets of equations can be formed for this purpose efforts were made to classify the various sets of equations, with the aim of gaining more physical content for the calculated angles. The numerical treatment was applied to a three-state diabatic potential matrix devised for the $\mathrm{Na}_{3}$ excited states (Cocchini, F.; Upton, T. H.; Andreoni, W. J. Chem. Phys. 1988, 88, 6068). A comparison between two-state and three-state angles reveals that, in certain cases, the two-state angles contain information regarding the interaction of the lower state with the upper states. However in general the twostate treatment may fail in yielding the correct topological features of the system. One of the main results of this study is that the adiabatic-diabatic transformation matrix, upon completion of a cycle, becomes diagonal again with the numbers \pm 1 in its diagonal.
\end{abstract}

\section{Introduction}

The need to consider effects due to higher electronic states (with respect to the ground state) may become important when one is interested in studying molecular processes in a given environment. Obviously, such effects are of major importance when these higher states interfere directly with the ground state as, for instance, in the case of charge transfer. ${ }^{1,2}$ Recently, however, it has become evident that molecular processes taking place on a given electronic state may be significantly affected by states that are far above that state. ${ }^{3-18}$ In particular, two recent studies in which results of single-surface and two-surface scattering processes were compared showed undoubtedly significant discrepancies. ${ }^{17,18}$ These studies also showed that singlestate results can be improved by employing an extended version of the ordinary Born-Oppenheimer (BO) single-state equation, ${ }^{17,19,20}$ which contains the nonadiabatic coupling terms that are responsible for the effects due to higher states. Such an extension can be performed in a pure two-state case (and eventually in some particular situations of multistate systems ${ }^{21}$ ). The immediate question to be asked is how to modify the extended single-state equation in case the two-state system is disturbed by a third state. ${ }^{22}$ This question deserves to be treated in separate studies and will not be considered here.

In the present article, we intend to get more familiar with the three-state case, with an emphasis on the adiabatic-diabatic

\footnotetext{
$\dagger$ Guest Professor at the University of Coimbra, P-3049 Coimbra Codex, Portugal.

$\doteqdot$ Guest Professor at the Institute of Atomic and Molecular Science, Tapei, Taiwan.
}

transformation (ADT) matrix. ${ }^{23}$ The ADT matrix is an orthogonal matrix responsible for the transformation from the adiabatic framework, characterized by dynamical nonadiabatic coupling terms, to the diabatic framework, characterized by potential coupling terms. This matrix, in fact, guarantees the inclusion of the correct topological effects in the nuclear (Schroedinger) equations as well as of the correct boundary conditions. ${ }^{19}$ Top and $\mathrm{Baer}^{24}$ suggested to express this matrix in terms of three angles somewhat reminiscent of the Euler angles, and they derived the differential equations for these angles. In a later publication, these equations ${ }^{25}$ were briefly analyzed. In what follows, the study of these angles is extended significantly. We shall consider various systems of differential equations and apply them to different physical situations. To obtain deeper insight, these equations will then be solved for a model potential to obtain the appropriate ADT angles. A comparison between the three-state angles and the corresponding two-state angles will be found to yield information on the way a third coupled state affects a two-state system.

\section{The General Approach}

II.1. Representation of the Adiabatic-Diabatic Transformation Matrix for a Three-State System: Derivation of the Differential Equations. One of the present authors showed that the ADT matrix A fulfills the following first-order differential equation: ${ }^{23}$

$$
\nabla A+\tau A=0
$$

where $\tau$ is a vector of matrices that contains the nonadiabatic 
coupling terms. In order for this system to have a unique and well-defined solution, the components of $\tau$ have to fulfill the following condition: ${ }^{23}$

$$
\operatorname{curl} \tau=[\tau \times \tau]
$$

This condition ensures the ability of $\tau$ to form diabatic potential matrix. As will be seen, our starting point is a diabatic matrix and therefore this condition is of formal importance only.

Presenting A as

$$
A=\left(\begin{array}{lll}
a_{11} & a_{12} & a_{13} \\
a_{21} & a_{22} & a_{23} \\
a_{31} & a_{32} & a_{3}
\end{array}\right)
$$

and recalling that $\tau$ is given in the form

$$
\tau=\left(\begin{array}{lll}
0 & \tau_{12} & \tau_{13} \\
-\tau_{12} & 0 & \tau_{23} \\
-\tau_{13} & -\tau_{23} & 0
\end{array}\right)
$$

(where $\tau_{i j}$ are vectors), we obtain, following the substitution of eqs 3 and 4 in eq 1, the explicit first-order (vector) equations for the various elements $a_{i k}: 24$

$$
\begin{aligned}
& \nabla a_{1 k}=-\tau_{12} a_{2 k}-\tau_{13} a_{3 k} \\
& \nabla a_{2 k}=\tau_{12} a_{1 k}-\tau_{23} a_{3 k} \quad k=1,2,3 \\
& \nabla a_{3 k}=\tau_{13} a_{1 k}+\tau_{23} a_{2 k}
\end{aligned}
$$

As is noticed, the elements of each column form a set of equations per se, independent of the rest. As it stands, nine equations are encountered. However, since $A$ is an orthogonal matrix only three of them are independent. Equations 5 can, therefore, be simplified significantly and this will be done next. To see how to do it we shall first consider a simplified case where $\tau$ is equal to $\tau^{(12)}$ defined as

$$
\tau^{(12)}=\left(\begin{array}{lll}
0 & \tau_{12} & 0 \\
-\tau_{12} & 0 & 0 \\
0 & 0 & 0
\end{array}\right)
$$

Substituting eq 6 in eq 1 produces for $A$ the ADT matrix $Q^{(12)}$ characterized by one ADT angle $\theta_{12}$,

$$
Q^{(12)}\left(\theta_{12}\right)=\left(\begin{array}{ccc}
\cos \theta_{12} & \sin \theta_{12} & 0 \\
-\sin \theta_{12} & \cos \theta_{12} & 0 \\
0 & 0 & 1
\end{array}\right)
$$

where $\theta_{12}$ fulfills the equation ${ }^{23}$

$$
\nabla \theta_{12}+\tau_{12}=0
$$

Similar matrices, namely $Q^{(23)}\left(\theta_{23}\right)$, and $Q^{(13)}\left(\theta_{13}\right)$ can be obtained when $\tau$ in eq 1 is replaced by $\tau^{(23)}$ and $\tau^{(13)}$, respectively. Thus, we find that each $\tau^{(i j)}$ matrix is characterized by one angle so we may assume that the general ADT matrix will be defined as a product of three matrices of the kind ${ }^{24}$

$$
A\left(\theta_{12}, \theta_{23}, \theta_{13}\right)=\prod_{i<j} Q^{(i j)}\left(\theta_{i j}\right)
$$

We already mentioned that the nine elements of an arbitrary three-dimensional orthogonal matrix can be expressed in terms of three independent angles. Therefore, this presentation is general and any solution based on eq 1 will be relevant to the problem under consideration.
In eq 9, we did not specify the order in which the multiplication is done, and since the product of two such matrices is not commutative, it is expected that each order will yield a different $A$ matrix. However, the $A$ matrix is a solution of eq 1 with given boundary conditions, and therefore it is uniquely determined and is in fact independent of the multiplication order in eq 9. Each given order will produce a different set of $\theta_{i j}$ angles. The systems of equations are different in each group due to the way the various $\tau_{i j}$ matrix elements enter the equations. For instance, in case the product order is $(12) \times(23) \times(13)$, eqs 5 yield the following equations for the three angles ${ }^{23}$

$$
\begin{gathered}
\nabla \theta_{12}=-\tau_{12}-\tan \theta_{23}\left(-\tau_{13} \cos \theta_{12}+\tau_{23} \sin \theta_{12}\right) \\
\nabla \theta_{23}=-\left(\tau_{23} \cos \theta_{12}+\tau_{13} \sin \theta_{12}\right) \\
\nabla \theta_{13}=-\left(\cos \theta_{23}\right)^{-1}\left(-\tau_{13} \cos \theta_{12}+\tau_{23} \sin \theta_{12}\right)
\end{gathered}
$$

Changing the positions of the two right-hand side matrices, namely, assuming the product order to be $(12) \times(13) \times(23)$, the equations have the form

$$
\begin{gathered}
\nabla \theta_{12}=-\tau_{12}-\tan \theta_{13}\left(\tau_{23} \cos \theta_{12}+\tau_{13} \sin \theta_{12}\right) \\
\nabla \theta_{13}=\tau_{23} \sin \theta_{12}-\tau_{13} \cos \theta_{12} \\
\nabla \theta_{23}=-\left(\cos \theta_{13}\right)^{-1}\left(\tau_{23} \cos \theta_{12}+\tau_{13} \sin \theta_{12}\right)
\end{gathered}
$$

It is easy to see that there are altogether six different ways of forming the $A$ matrix from the product of the three different $\mathrm{Q}^{(i j)}\left(\theta_{i j}\right)$ matrices. This group is made up of two subgroups, each containing three different products related to each other by cyclic permutations. One group contains the products: $(12) \times(23) \times$ $(13),(23) \times(13) \times(12)$, and $(13) \times(12) \times(23)$ and the other the products $(12) \times(13) \times(23),(13) \times(23) \times(12)$, and $(23)$ $\times(12) \times(13)$. The general set of equations for the first group can be shown to be

$$
\begin{gathered}
\nabla \theta_{i j}=-\tau_{i j}-\tan \theta_{j k}\left(\tau_{j k} \sin \theta_{i j}-\tau_{i k} \cos \theta_{i j}\right) \\
\nabla \theta_{j k}=-\left(\tau_{i k} \sin \theta_{i j}+\tau_{j k} \cos \theta_{i j}\right) \\
\nabla \theta_{i k}=-(-1)^{p}\left(\cos \theta_{j k}\right)^{-1}\left(\tau_{j k} \sin \theta_{i j}-\tau_{i k} \cos \theta_{i j}\right)
\end{gathered}
$$

and the general set of equations for the second group can be shown to be

$$
\begin{gathered}
\nabla \theta_{i j}=-\tau_{i j}-\tan \theta_{i k}\left(\tau_{j k} \cos \theta_{i j}+\tau_{i k} \sin \theta_{i j}\right) \\
\nabla \theta_{i k}=\tau_{j k} \sin \theta_{i j}-\tau_{i k} \cos \theta_{i j} \\
\nabla \theta_{j k}=-(-1)^{p}\left(\cos \theta_{i k}\right)^{-1}\left(\tau_{j k} \cos \theta_{i j}+\tau_{i k} \sin \theta_{i j}\right)
\end{gathered}
$$

where $p=0$ is for the first and the third products and $p=1$ is for the second product (in each subgroup). In these equations, $\theta_{i j}$ is the angle of the left-hand side matrix, $\theta_{j k}$ is the angle of the central matrix, and $\theta_{i k}$ is the angle of the right-hand matrix. Thus, the order of the matrices in the case of eqs 11a is (ij)$(j k)(i k)$. In the case of eqs $11 \mathrm{~b}$, it is also $(i j)(j k)(i k)$, but here $(j k)$ stands for $(i k)$ in the previous case, etc.

So far we have discussed six different ways to form the $A$ matrix. In fact there are more ways, for instance, presenting $A$ as the product $Q^{(12)}\left(\theta_{12}\right) Q^{(13)}\left(\theta_{13}\right) Q^{(12)}\left(\theta_{23}\right)$ (this matrix is identical to the Euler rotation matrix for a rigid body) is also perfectly legitimate but leads to a different set of equations. However, the solution of such a system of equation can be 
obtained by solving one system of equations belonging to the first group with appropriate boundary conditions.

II.2. Analysis of the Equations and Some of Their Solutions. Three features characterize the newly derived system of equations.

(1) We found that irrespective of the order of the matrices it is always the equation for $\theta_{i j}$, namely, the angle of the lefthand side matrix in the product, that contains (the corresponding) $\tau_{i j}$ element as a free term. This guarantees that when the two other $\tau$ elements become zero, the three-state case reduces smoothly to a two-state case, with the relevant equation for the one left angle (see eq 8). This form is also convenient in case $\tau_{i j}$ is the dominating nonadiabatic coupling term and the other two $\tau$ 's are of second-order only. Another interesting situation is encountered when one of the two $\tau$ matrix elements, $\tau_{j k}$ and $\tau_{i k}$, is much smaller than the other as well as much smaller than $\tau_{i j}$ itself. So let us consider eqs $11 \mathrm{a}$ with the boundary conditions $\theta_{i j}(\varphi=0)=0, \theta_{i k}(\varphi=0)=0, \theta_{j k}(\varphi=0)=0$, and assume $\tau_{i k}$ $\ll \tau_{i j}, \tau_{j k}$. Since, in the equation for $\theta_{i j}$ the large $\tau_{j k}$ is multiplied by $\sin \theta_{i j}$ (which is 0 at the beginning of the integration) and the small $\tau_{i k}$ is multiplied by $\cos \theta_{i j}$ (which is 1 at the beginning of the integration) the overall rate of change of $\theta_{i j}$ is determined mainly by $\tau_{i j}$ itself, just as in the two-state case. In our numerical example, we discuss a situation like that and we shall show that some three-state features are reproduced within the approximated two-state model.

(2) We also found that within each system of equations, out of the three equations only two are coupled. These two equations are related to the two angles of the left-hand side matrices, whereas the (third) angle belonging to the right-hand matrix can be derived by a simple integration once the first two become available. This feature may, in certain cases, help to identify the kind of interaction a strongly coupled two-state system has with a loosely coupled third state.

(3) So far, we have assumed that the integration of any system of the equations will be done with the boundary conditions $\left(\theta_{i j}{ }^{-}\right.$ $\left.(\varphi=0)=0, \theta_{i k}(\varphi=0)=0, \theta_{j k}(\varphi=0)=0\right)$. In fact, this is not necessary and we may choose any set of boundary conditions. In this respect, it can be shown analytically that solving eqs $10 \mathrm{a}$ with the initial conditions $\left(\theta_{12}(\varphi=0)=\pi / 2, \theta_{23}(\varphi=0)=\right.$ $0, \theta_{13}(\varphi=0)=0$ ) yields a solution identical to a solution of eq $10 \mathrm{~b}$ with the initial conditions $\left(\theta_{12}(\varphi=0)=0, \theta_{23}(\varphi=0)=0\right.$, $\left.\theta_{13}(\varphi=0)=0\right)$. The only difference is that the values of $\theta_{12}$ are shifted by $\pi / 2$. As a result, the system of equations in eq $10 \mathrm{~b}$ becomes, in fact, redundant. This finding can be generalized to any set of angles, namely, $\left(\theta_{i j}(\varphi=0)=\pi / 2, \theta_{i k}(\varphi=0)=0\right.$, $\left.\theta_{j k}(\varphi=0)=0\right)$ and therefore it implies that the whole group of systems of equations in eqs $11 \mathrm{~b}$ is redundant as well.

From now on we shall refer to solutions of equations belonging to (11a) (or 10a) only.

\section{Three-State Model}

The three-state model we propose to consider is a model potential suggested by Cocchini et al. ${ }^{26}$ to study the excited states of $\mathrm{Na}_{3}$. Since our way to present this potential matrix is different, we shall derive it next. We start with the $2 \times 2$ potential matrix, $V^{(2)}$, which we obtained some time ago $\mathrm{o}^{27,20}$ and which is closely related to a potential matrix devised by Longuet-Higgins to study the $\mathrm{e} \otimes \mathrm{E}$ interaction. ${ }^{28,29}$ It is of the form

$$
V^{(2)}=\left(\begin{array}{cc}
\epsilon_{\mathrm{E}}+U_{1} & U_{2} \\
U_{2} & \epsilon_{\mathrm{E}}-U_{1}
\end{array}\right)
$$

where $\epsilon_{\mathrm{E}}$ is an E-type state and $U_{i}, i=1,2$, are defined as

$$
U_{1}=k \rho \cos \varphi+\frac{1}{2} g \rho^{2} \cos (2 \varphi)
$$

and

$$
U_{2}=k \rho \sin \varphi-\frac{1}{2} g \rho^{2} \sin (2 \varphi)
$$

Here, $\rho$ and $\varphi$ are polar nuclear coordinates and $k$ and $g$ are characteristic coupling parameters. Next, we extend this model potential to describe a three-state system by treating the following $3 \times 3$ potential matrix $V^{(3)}$ :

$$
V^{(3)}=\left(\begin{array}{ccc}
\epsilon_{\mathrm{E}}+U_{1} & U_{2} & W_{1}-W_{2} \\
U_{2} & \epsilon_{\mathrm{E}}-U_{1} & W_{1}+W_{2} \\
W_{1}-W_{2} & W_{1}+W_{2} & \epsilon_{\mathrm{A}}
\end{array}\right)
$$

Here, $\epsilon_{\mathrm{E}}$ and the $U_{i}, i=1,2$, potentials are as before, $\epsilon_{\mathrm{A}}$ is the value of another electronic state, and $W_{i}, i=1,2$, are potentials of the same form as the $U_{i}$ 's but defined in terms of a different set of parameters $f$ and $p$, which replace $k$ and $g$, respectively. This particular form was chosen for two reasons: (1) In case $W_{i} \equiv 0, i=1,2$, the $V^{(3)}$ matrix reduces to the $V^{(2)}$ potential and (2) in the case $U_{i} \equiv 0, i=1,2$ the model produces the pseudoJahn-Teller (PJT) model ${ }^{22,26}$ with the following eigenvalues:

$$
\begin{aligned}
& \lambda_{1}=\epsilon_{\mathrm{E}} \\
& \lambda_{2}=\epsilon_{0}+\sqrt{(\Delta \epsilon)^{2}+2\left(W_{1}^{2}+W_{2}^{2}\right)} \\
& \lambda_{3}=\epsilon_{0}-\sqrt{(\Delta \epsilon)^{2}+2\left(W_{1}^{2}+W_{2}^{2}\right)}
\end{aligned}
$$

where $\epsilon_{0}$ and $\Delta \epsilon$ are defined as

$$
\epsilon_{0}=\frac{1}{2}\left(\epsilon_{\mathrm{A}}+\epsilon_{\mathrm{E}}\right) \quad \text { and } \quad \Delta \epsilon=\frac{1}{2}\left(\epsilon_{\mathrm{A}}-\epsilon_{\mathrm{E}}\right)
$$

The eigenvalues presented in eq 15 are identical to those of Cocchini et al. ${ }^{26}$ in a similar situation (i.e., for the PJT model).

\section{Numerical Study}

In the previous section, a potential was presented in terms of two polar coordinates $\rho$ and $\varphi$. Our numerical study will concentrate on the coordinate $\varphi$ that is defined along the interval $(0,2 \pi)$, and therefore, in all what follows the continuous variable will be $\varphi$. The coordinate $\rho$, defined as the radial distance with respect to the point of (the main) degeneracy, will be considered as a parameter, but results will be presented for several $\rho$ values. A second parameter that we shall frequently use is the potential energy shift, $\Delta \epsilon$, introduced in eq 16. $\Delta \epsilon$ is the shift between the two original adiabatic states and the third adiabatic state at the origin, i.e., at $\rho=0$ (in case $\Delta \epsilon=0$ all three states are degenerate at the origin) and results will be presented for several of its values. In addition to these parameters, we also assign numerical values to $k$ and $g$ to define $U_{1}$ and $U_{2}$ (see eqs 13 ), and $p$ and $f$ to define $W_{1}$ and $W_{2}$. The actual values were taken from Cocchini et al. ${ }^{26}$ who used them to assign the excited state spectrum for gas-phase $\mathrm{Na}_{3}$ in the energy region $<2.7 \mathrm{eV}$,

$$
k=\sqrt{2} p=5.53 \mathrm{au} \text { and } g=\sqrt{2} f=0.152 \mathrm{au}
$$




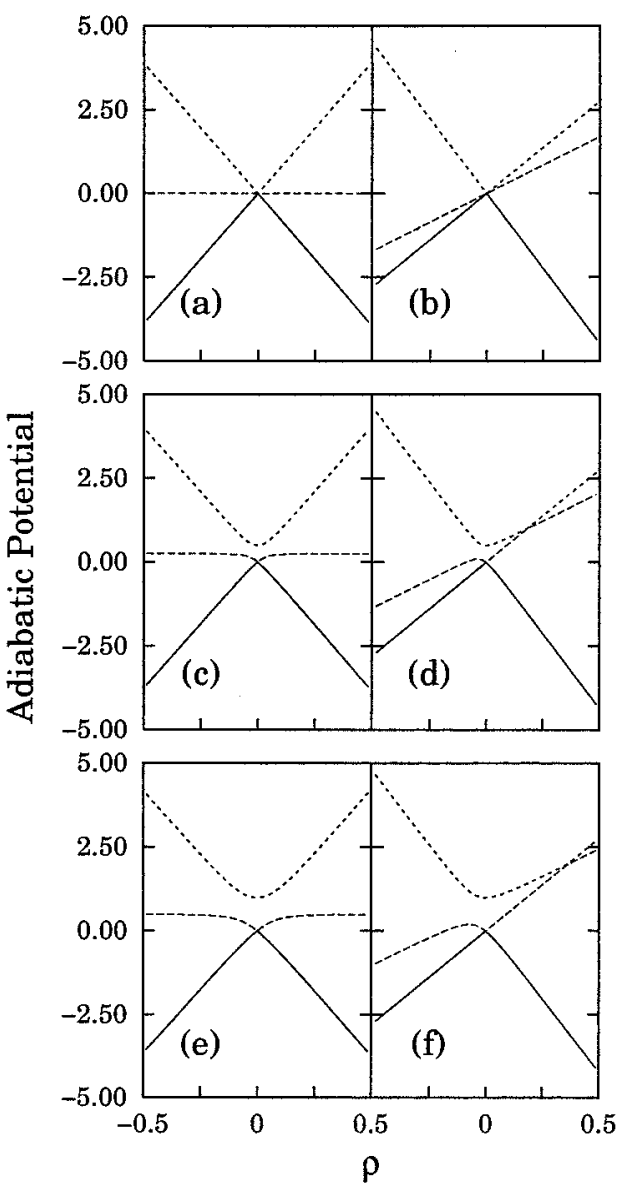

Figure 1. Adiabatic potential energy curves (obtained for the model potential described in section III) as a function $\rho$ calculated for different $\varphi$ and $\Delta \epsilon$ values. The values for negative $\rho$ are those calculated for positive $\rho$ but with an opposite angle (i.e., $\varphi+\pi$ ): (a) $\Delta \epsilon=0, \varphi=$ 0 ; (b) $\Delta \epsilon=0, \varphi=\pi / 2$; (c) $\Delta \epsilon=0.25, \varphi=0$; (d) $\Delta \epsilon=0.25, \varphi=$ $\pi / 2$; (e) $\Delta \epsilon=0.5, \varphi=0$; (f) $\Delta \epsilon=0.5, \varphi=\pi / 2$. The various lines refer to different adiabatic states.

In the numerical treatment we concentrate mainly on the $\varphi$ dependence of the various ADT angles as will be introduced in the following subsections, but we shall first present the $\varphi$ and $\rho$ dependence of the adiabatic potential energy surfaces and of the nonadiabatic coupling terms.

IV.1. Adiabatic Potential Energy Surfaces. In Figures 1 and 2 are shown the three adiabatic potential energy surfaces of this system; in Figure 1 are shown $\rho$-dependent curves as calculated for the two mirror angles once for $\varphi=(0, \pi)$ and once for $\varphi=(\pi / 2,3 \pi / 2)$, and in Figure 2 the $\varphi$-dependent curves as calculated for different $\rho$ values. In both figures, the potential curves were calculated for different $\Delta \epsilon$ values.

From Figure 1a (the $\Delta \epsilon=0$ case), it is not clear what kind of a degeneracy is to be expected at $\rho=0$, namely, whether it is a triple, a double, or a single degeneracy. However, as $\Delta \epsilon$ increases, the surfaces separate and the situation clears up. In the case of $\varphi=(0, \pi)$, we encounter for all subsequent $\Delta \epsilon$ values (parts $\mathrm{c}$ and e of Figure 1) only one point of degeneracy (between surfaces 1 and 2), but in the case of $\varphi=(\pi / 2,3 \pi / 2)$, for the same $\Delta \epsilon$ 's two points of degeneracy are encountered, one point as before (at the origin) and a second point between surfaces 2 and 3 which occurs for each $\Delta \epsilon$ at a different $\rho$ value but for $\varphi=\pi / 2$ only. Similar points of degeneracy were obtained for $\varphi=7 \pi / 6$ and $\varphi=11 \pi / 6$ at the same $\rho$ value. Thus, for a given $\Delta \epsilon(\neq 0)$ we encounter, in addition to the degeneracy point at the origin, three points of degeneracy at
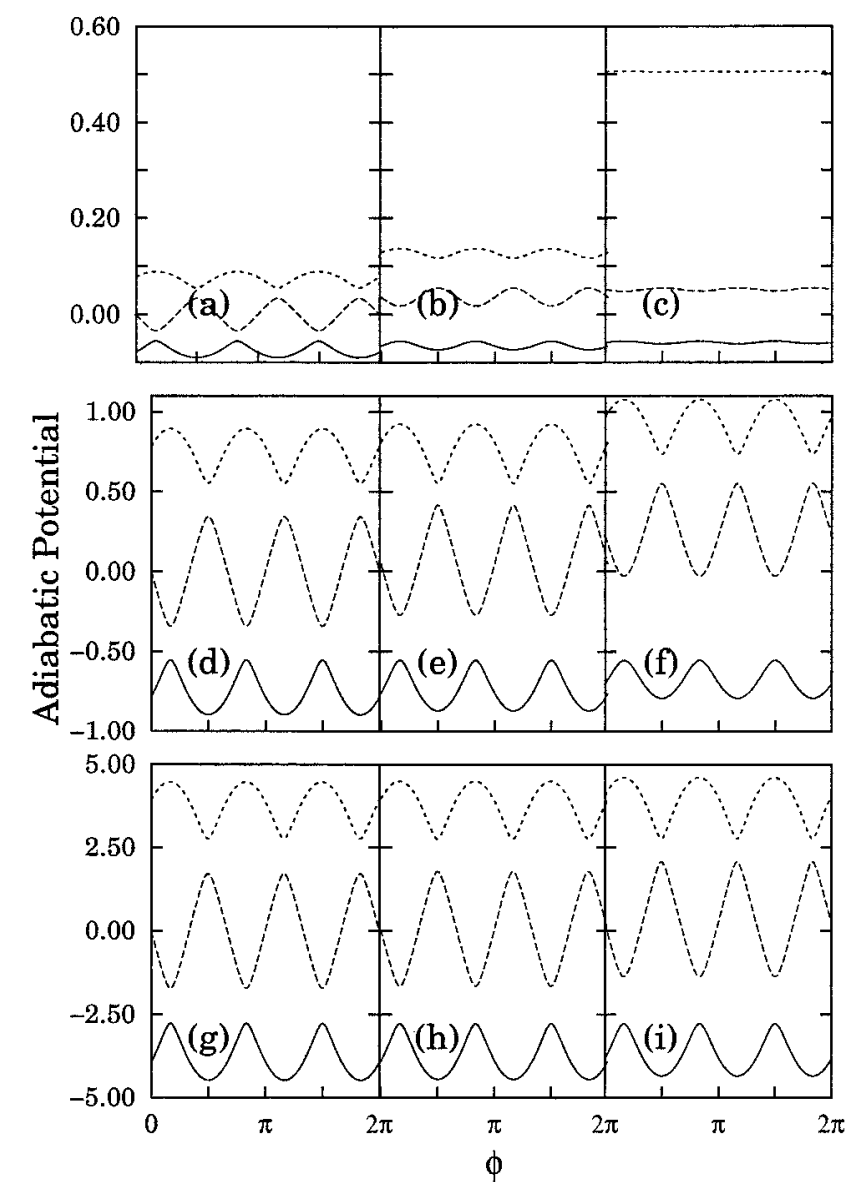

Figure 2. Adiabatic potential energy curves (obtained for the model potential described in section III) as a function $\varphi$ calculated for different values of $\rho$ and $\Delta \epsilon$ : (a) $\rho=0.01, \Delta \epsilon=0$; (b) $\rho=0.01, \Delta \epsilon=0.05$; (c) $\rho=0.01, \Delta \epsilon=0.25$; (d) $\rho=0.1, \Delta \epsilon=0$; (e) $\rho=0.1, \Delta \epsilon=0.05$; (f) $\rho=0.1, \Delta \epsilon=0.25$; (g) $\rho=0.5, \Delta \epsilon=0$; (h) $\rho=0.5, \Delta \epsilon=0.05$; (i) $\rho=0.5, \Delta \epsilon=0.25$. The various lines refer to different adiabatic states.

the above-mentioned three fixed angles (see also ref 22). Therefore, when a fixed $\rho$-circle surrounds the origin, it contains either one point of degeneracy (at the origin) or four points of degeneracy. This situation is reminiscent of the "linear plus quadratic $\mathrm{e} \otimes \mathrm{E}$ two-state model" treated on several occasions. ${ }^{20,28,29}$

Returning now to the case of $\Delta \epsilon=0$ it is obvious that at the origin we have two degeneracy points: one formed between the first and the second states and one between the second and the third states (the number of degenerate points at a given point in configuration space cannot be more than 3 because we have only a $3 \times 3$ matrix).

In Figure 2 are presented the $\varphi$-dependent potentials as calculated for fixed $\rho$ values and for several values of $\Delta \epsilon$. In general, the potential has a wavy structure with three maxima and three minima. It is noticed that each surface is close to being the mirror image of the surface adjacent to it. For a given $\rho$ value, the amplitude of the wavy structure decreases as $\Delta \epsilon$ increases, but the rate of decrease is relatively slow. It is also noted that the maxima points of second surface face the minima points of the third surface at the above-mentioned $\varphi$ angles, namely, $\varphi=\pi / 2,7 \pi / 6$, and $11 \pi / 6$.

IV.2. Nonadiabatic Coupling Terms. The diabatic potential matrix given in eq 14 was used to calculate the three nonadiabatic coupling terms $\tau_{s i j}, i, j=1,2,3(i \neq j)$, where $s$ is a nuclear coordinate. This we do by employing the Hellmann-Feynmann 


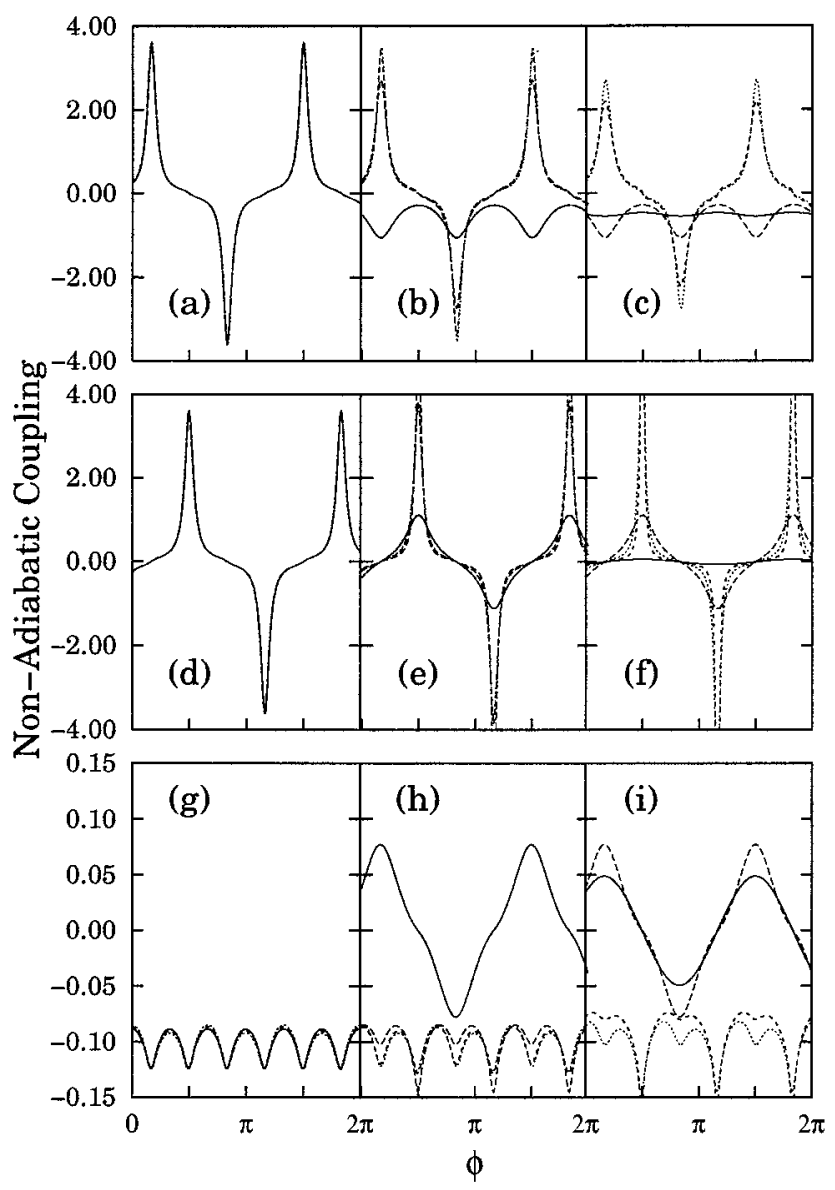

Figure 3. The three nonadiabatic coupling terms (obtained for the model potential described in section III) as a function $\varphi$ calculated for different values of $\rho$ and $\Delta \epsilon$ : (a) $\tau=\tau_{12}, \Delta \epsilon=0.0$; (b) $\tau=\tau_{12}, \Delta \epsilon=$ 0.05 ; (c) $\tau=\tau_{12}, \Delta \epsilon=0.5$; (d) $\tau=\tau_{23}, \Delta \epsilon=0.0$; (e) $\tau=\tau_{23}, \Delta \epsilon=$ 0.05 ; (f) $\tau=\tau_{23}, \Delta \epsilon=0.5$; (g) $\tau=\tau_{13}, \Delta \epsilon=0.0$; (h) $\tau=\tau_{13}, \Delta \epsilon=$ 0.05 ; (i) $\tau=\tau_{13}, \Delta \epsilon=0.5$; (-) $\rho=0.01,(---) \rho=0.1$; (- -) $\rho$ $=0.5 ;(\cdots) \rho=1.0$.

theorem, namely,

$$
\tau_{s i j}=\frac{\left\langle\psi_{i}\left|\frac{\partial V}{\partial s}\right| \psi_{j}\right\rangle}{\lambda_{i}-\lambda_{j}}
$$

where $\psi_{i}\left(\psi_{j}\right)$ is an eigenvector of the matrix $V$. In the forthcoming discussion, the coordinate $\mathrm{s}$ will be identified with $\varphi$. In Figure 3 are presented the three nonadiabatic coupling terms $\tau_{\varphi i j}, i, j=1,2,3(i>j)$, as calculated for different values of $\rho$ and $\Delta \epsilon$. The following features are to be noticed.

(1) It is seen that $\tau_{12}$ and $\tau_{23}$, namely, the terms responsible for the coupling between the two adjacent states, are much stronger than $\tau_{13}$, the (nonadiabatic) coupling term between the two nonadjacent states. Therefore, the main interaction between the two-state system and the third state is due to its coupling with the second state. This is in accordance with what was discussed earlier regarding the $\rho$-dependent potential curves.

(2) In general, $\tau_{12}$ and $\tau_{23}$ are only weakly dependent on both $\Delta \epsilon$ and $\rho$. Usually, the shape is a tripeak function. This is so for $\Delta \epsilon=0$, where we have at the origin a three-state degeneracy (and no other points of degeneracy) and in the case $\Delta \epsilon \neq 0$ but $\rho$ large enough. In this case, the fixed- $\rho$ path encircles (as was discussed earlier) one point of degeneracy, between the two lowest states, located at the origin and three points of degeneracy between the second and the third states located at some distance

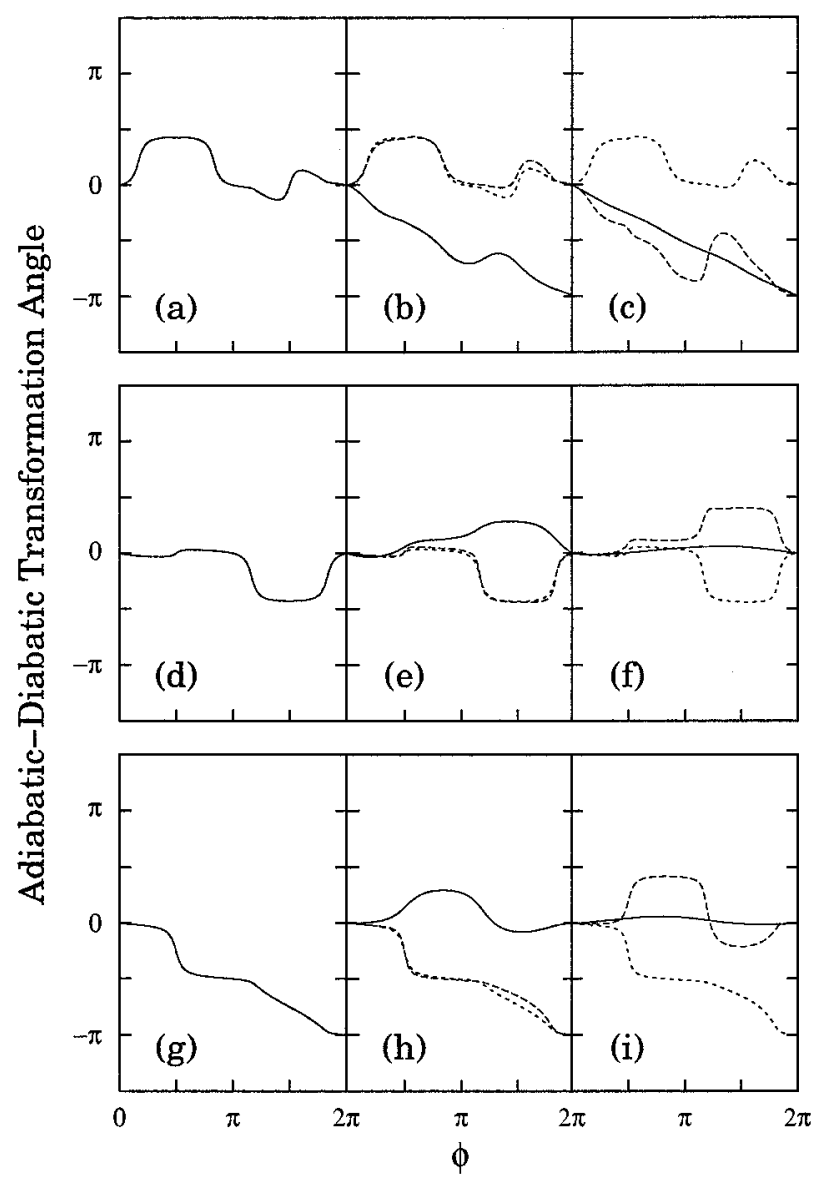

Figure 4. The three adiabatic-diabatic transformation angles (obtained by solving eqs 10a) as a function $\varphi$ calculated for different values of $\rho$ and $\Delta \epsilon$ : (a) $\theta=\theta_{12}, \Delta \epsilon=0.0$; (b) $\theta=\theta_{12}, \Delta \epsilon=0.05$; (c) $\theta=\theta_{12}$, $\Delta \epsilon=0.25$; (d) $\theta=\theta_{23}, \Delta \epsilon=0.0$; (e) $\theta=\theta_{23}, \Delta \epsilon=0.05$; (f) $\theta=\theta_{23}$, $\Delta \epsilon=0.25$; (g) $\theta=\theta_{13}, \Delta \epsilon=0.0$; (h) $\theta=\theta_{13}, \Delta \epsilon=0.05$; (i) $\theta=\theta_{13}$, $\Delta \epsilon=0.25$; (-) $\rho=0.01 ;(---) \rho=0.1 ;(--) \rho=0.5$.

from the origin. It is only when $\Delta \epsilon$ is large enough and $\rho$ relatively small that $\tau_{12}$ and $\tau_{23}$ attain their expected two-state values. Thus, $\tau_{12} \rightarrow 1 / 2$ because a pure conical intersection situation is encountered in this case between the first and the second states ${ }^{27}$ and $\tau_{23} \rightarrow 0$ because no degeneracy is found in this situation between the second and the third states (see Figure 1 ). As for $\tau_{13}$, it is seen to have only relatively small values because no degeneracy exists between the first and the third state. An interesting point to emphasize here is that $\tau_{13}$ reveals the fact that even for $\Delta \epsilon=0$ we do not have a degeneracy between the first and the third states, not at the origin and not at any other point.

(3) The three-peak $\varphi$ dependence of the two leading $\tau$ functions is closely associated with the wave-type structure of the electronic eigenvalues, as presented in Figure 2. It is noticed that the peaks appear at the same points where the two respective states get closest to each other.

IV.3. Adiabatic-Diabatic Transformation Angles. Each system of equations yields three angles, one angle for each matrix in eq 9. It is important to mention that this is the first time that these equations are solved numerically. The relevance of the solutions was tested by comparing the final ADT matrices, as obtained from solving the six different systems of equations. In all our tests the calculated ADT matrices were exactly identical immaterial which system of equations was used.

In Figure 4 are presented the $\varphi$-dependent angles as calculated from eqs 10a for different values of $\rho$ and $\Delta \epsilon$. The main features 
to be noticed are as follows. (a) In all studied cases three angles attain, upon completion of a cycle, either the value $-\pi$ (in what follows we shall not distinguish between $\pi$ and $-\pi$ ) or zero. (b) It is always that one angle attains the value of $\pi$ and the two others the value of zero. (c) The angle $\theta_{23}$ always ends up with the value of zero. (d) In the cases that $\Delta \epsilon \neq 0$ and $\rho$ is small enough the angle $\theta_{12}$ decreases linearly to $-\pi$ and the two others are, essentially, flat zero functions. (e) In all other cases $\theta_{12}$ and $\theta_{23}$ are oscillatory functions of $\varphi$, while $\theta_{13}$ decreases uniformly to $-\pi$.

Not all features seen here can be explained without performing more detailed studies. In what follows, we just refer to a few of them. The feature mentioned first is most interesting but above all the most important one. Like in the two-state case where the ADT angle attains, upon completion of a cycle, either the value of $\pi$ (the Jahn-Teller conical intersection (CI) case) or zero (for the quadratic Renner-Teller situation), here too, the final values of the ADT angles are either $\pi$ or zero. However, since we always have two angles that become zero and one that becomes $\pi$, the ADT matrix, upon completion of a cycle, will contain two $(-1)$ 's and one $(+1)$ in the diagonal. At this stage, it is important to mention that we recently managed to prove analytically that an ADT matrix can yield diabatic states if and only if it becomes diagonal upon completion of a cycle, where the diagonal elements are $\pm 1 .{ }^{30}$ This is, indeed, the situation in our case. Since we started with a diabatic potential matrix, the ADT matrices obtained from it must have \pm 1 's in the diagonal upon completion of a cycle.

The second subject in this respect is the behavior of $\theta_{12}$ when $\Delta \epsilon \neq 0$ and $\rho$ are small enough. This is the situation when $\theta_{12}$ stops to be oscillatory and attains its linear decreasing shape (parts b and c of Figure 4). It is seen that the tendency to become linear enhances as $\Delta \epsilon$ becomes larger. In fact, we find for a given $\rho$ the following relation:

$$
\lim _{\Delta \epsilon \rightarrow \infty} \theta_{12} \rightarrow \frac{1}{2} \varphi
$$

which is characteristic of the two-state conical intersection situation. This behavior is expected because, as described in section IV.1 (see also Figure 1), when $\Delta \epsilon$ becomes large enough a fixed $\rho$-circle surrounds a single isolated conical intersection located at the origin. In all other cases, the fixed $\rho$ circle surrounds an even number of points of degeneracy. For $\Delta \epsilon=$ 0 , we have a double degeneracy at the origin (as discussed earlier) and therefore this angle is expected to be zero, and in the case when $\Delta \epsilon \neq 0$ and $\rho$ is large enough, the fixed $\rho$ circle surrounds four points of degeneracy (also, as discussed earlier) and so, again, it becomes zero.

The angle $\theta_{23}$ does not contain any information regarding the points of degeneracy located within a fixed $\rho$ circle (see parts $\mathrm{d}-\mathrm{f}$ of Figure 4$)$. It is either a flat zero function or a function that is close to zero along most of the $\varphi$ interval.

The angle $\theta_{13}$ exhibits a kind of "orthogonal" behavior to $\theta_{12}$. It become $\pi$ when $\theta_{12}$ becomes zero and vice-versa. This implies that the information delivered by $\theta_{13}$ is not complete because the effect of one point of degeneracy is missing (it is the one between the first and the second states). Thus, in the case when $\Delta \epsilon=0$, the angle $\theta_{13}$ "notices" only one degeneracy although there are two, in the case that $\Delta \epsilon \neq 0$ and $\rho$ is small, no degeneracy is detected although there is one point of degeneracy, and in the case of $\Delta \epsilon \neq 0$ and large $\rho$ values, it detects three points of degeneracy instead of four.

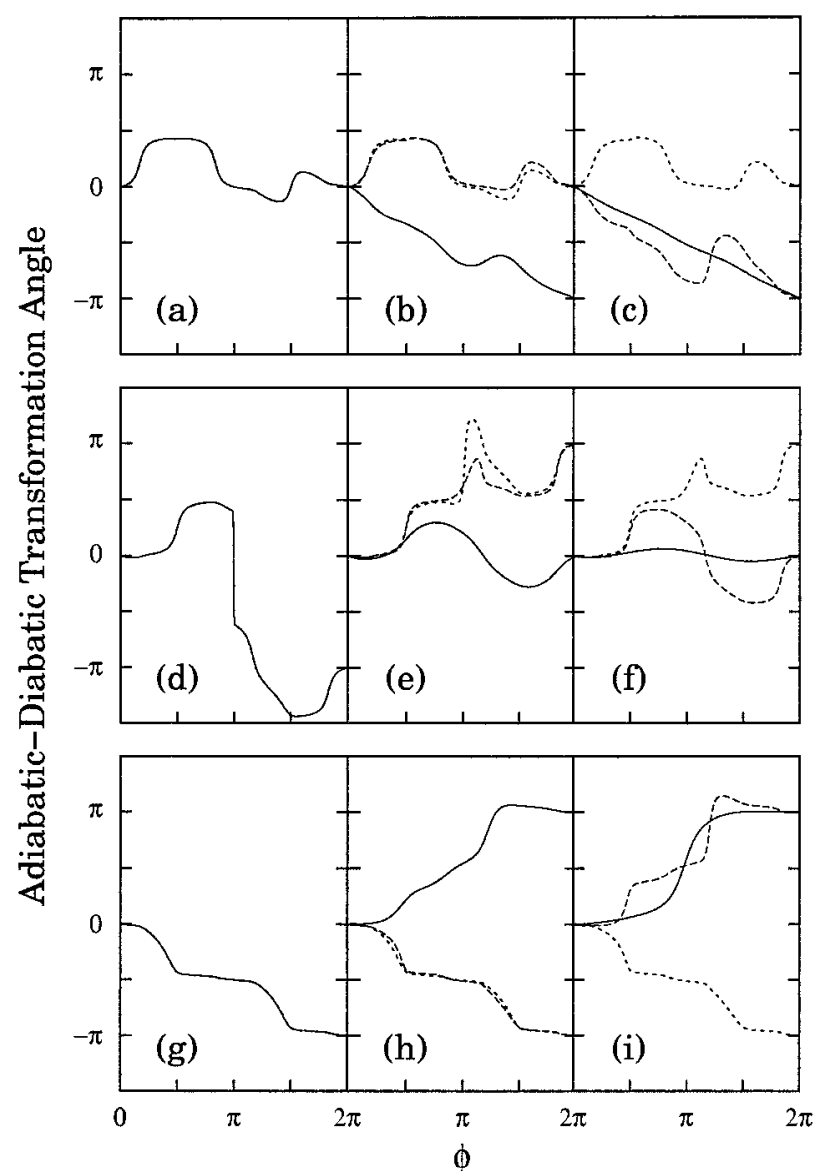

Figure 5. The three principal adiabatic-diabatic transformation angles (as obtained by solving eqs $11 \mathrm{a}$ each time with the corresponding $\tau_{i j}$ as the free leading term) as a function $\varphi$ calculated for different values of $\rho$ and $\Delta \epsilon$ : (a) $\theta=\theta_{12}, \Delta \epsilon=0.0$; (b) $\theta=\theta_{12}, \Delta \epsilon=0.05$; (c) $\theta=$ $\theta_{12}, \Delta \epsilon=0.25$; (d) $\theta=\theta_{23}, \Delta \epsilon=0.0$; (e) $\theta=\theta_{23}, \Delta \epsilon=0.05$; (f) $\theta$ $=\theta_{23}, \Delta \epsilon=0.25$; (g) $\theta=\theta_{13}, \Delta \epsilon=0.0$; (h) $\theta=\theta_{13}, \Delta \epsilon=0.05$; (i) $\theta=\theta_{13}, \Delta \epsilon=0.25$; (-) $\rho=0.01 ;(---) \rho=0.1$; (- - ) $\rho=0.5$.

Next are considered the three leading angles (henceforth termed the "principal" angles), which are the $\theta_{i j}$ angles calculated from their "own" system of equations. Thus, the principal angle $\theta_{\mathrm{p} 12}$ is obtained from system of equations where $\tau_{12}$ is the (free) leading term (in the present case $\theta_{12}$ and $\theta_{\mathrm{p} 12}$ are identical), the principal angle $\theta_{\mathrm{p} 23}$ is obtained from the system of equations where $\tau_{23}$ is the leading term, and the same applies for $\theta_{\mathrm{p} 13}$. In Figure 5 are presented the $\varphi$-dependent principal angles as calculated from eqs 11a for different values of $\rho$ and $\Delta \epsilon$. The subfigures for $\theta_{\mathrm{p} 12}$ (parts a-c of Figure 5 are identical to the corresponding ones of Figure 4 and are presented here, again, for the sake of completeness). The angles $\theta_{\mathrm{p} 23}$ and $\theta_{\mathrm{p} 13}$ are seen to behave differently from the corresponding angles $\theta_{23}$ and $\theta_{13}$ shown in Figure 4 . As for $\theta_{\mathrm{p} 23}$, it exhibits much more "activity" than $\theta_{23}$ and seems to contain more information regarding the degeneracy points. But, like $\theta_{13}$ in Figure 4 , it always misses the effect of the degeneracy between the first and the second states. A somewhat "strange" behavior is presented by $\theta_{\mathrm{p} 13}$ (see parts $\mathrm{g}-\mathrm{i}$ of Figure 5). which is seen to contain more information than $\theta_{13}$. It usually misses the effect of one degeneracy except for cases when the fixed- $\rho$ circles surround the one single isolated point of degeneracy.

IV.4. Comparison between the Two-State and the ThreeState Adiabatic-Diabatic Transformation Angles. The last subject to be discussed is the comparison between the present three-state $\theta_{12}$ angle and the parallel two-state $\theta_{\mathrm{t} 12}$ angle obtained 

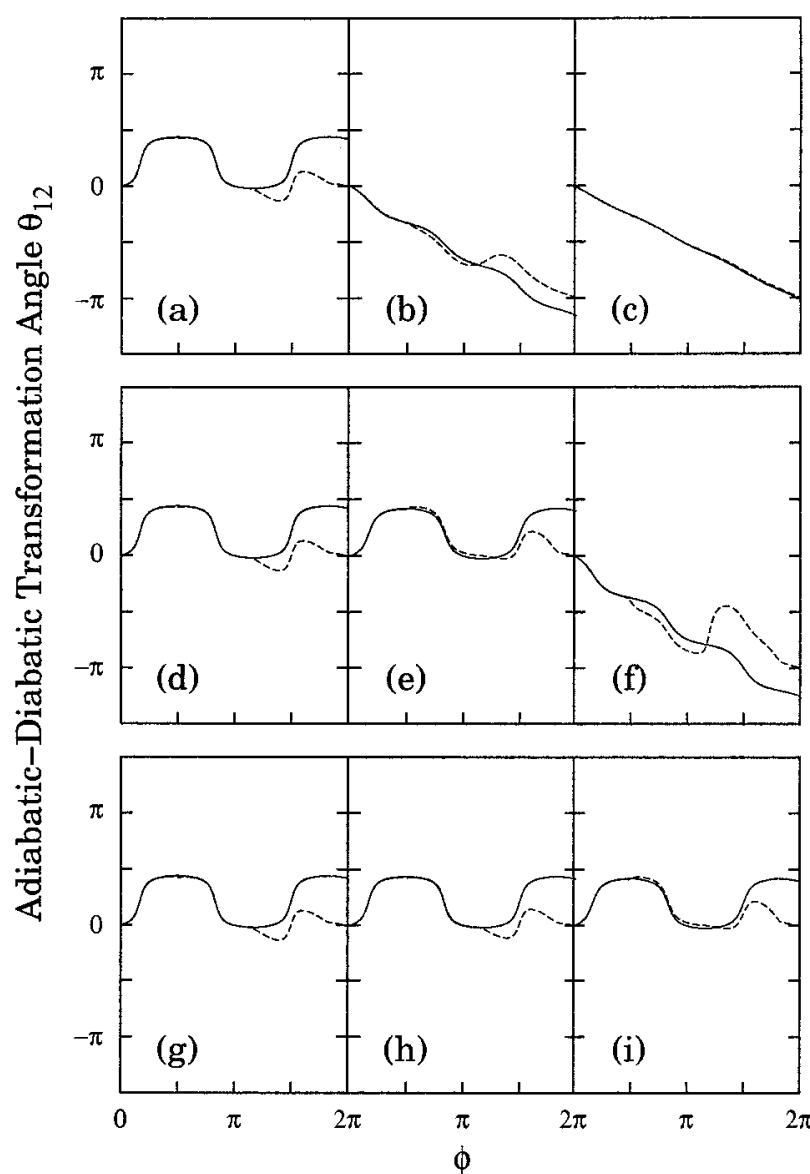

Figure 6. A comparison between a two-state and principal three-state $\theta_{12}$ values as a function $\varphi$ calculated for different values of $\rho$ and $\Delta \epsilon$ : (a) $\rho=0.01, \Delta \epsilon=0.0$; (b) $\rho=0.01, \Delta \epsilon=0.05$; (c) $\rho=0.01, \Delta \epsilon=$ 0.25 ; (d) $\rho=0.1, \Delta \epsilon=0.0$; (e) $\rho=0.1, \Delta \epsilon=0.05$; (f) $\rho=0.1, \Delta \epsilon$ $=0.25$; (g) $\rho=0.5, \Delta \epsilon=0.0$; (h) $\rho=0.5, \Delta \epsilon=0.05$; (i) $\rho=0.5, \Delta \epsilon$ $=0.25$; (-) two-state $\theta_{12},(--)$ principal three-state $\theta_{12}$.

by a straightforward integration over the $\tau_{12}(\varphi) .{ }^{23}$ Thus,

$$
\theta_{\mathrm{t} 12}=\int_{0}^{\varphi} \tau_{12}\left(\varphi^{\prime}\right) \mathrm{d} \varphi^{\prime}
$$

In Figure 6 are presented the two functions for various parameters. It seems that overall the fit between $\theta_{12}$ and $\theta_{\mathrm{t} 12}$ is, indeed, very encouraging. This holds in particular when $\rho$ is small enough and $\Delta \epsilon \neq 0$. In this case, the two angles are characteristic for a pure $\mathrm{CI}$ case as was discussed earlier. In all other cases, none of the two $\theta_{12}$ functions show a linear dependence on $\varphi$ which is an interesting result, in particular, for $\theta_{\mathrm{t} 12}$. This situation is reminiscent of several cases discussed by Yarkony and co-workers. ${ }^{31,32}$ They calculated from first principles the nonadiabatic coupling terms between the two lowest electronic states for a series of systems, i.e., $\mathrm{H}_{3}, \mathrm{H}_{2} \mathrm{~S}$, $\mathrm{CH}_{2},{ }^{31}$ and $\mathrm{AlH}_{2}{ }^{32}$ and then used eq 19 to calculate the ADT angles with the aim of deriving the topological (Berry) phase (namely, the ADT angle at $\varphi=2 \pi$ ). They found that as long as $\rho$ is not too large, the topological phase is, indeed, $\pi$, like in the present case, but when $\rho$ increases further the topological phase starts to decrease and the larger $\rho$ the larger the deviation from $\pi$.

The two functions behave very similarly also in the other cases, along most of the $\varphi$ range. However, in these cases, it is always toward the end of the $\varphi$ range that the two functions go apart. In this sense, $\theta_{\mathrm{t} 12}$ fails to reach the correct topological value. It usually ends up with a value close to $\pi / 2$ instead of zero. Here we would like to refer again to Yarkony's calculations. He attributed the failure of eq 19 to deliver the correct topological phase, (once $\rho$ becomes large) to the fact that the theory behind the connection between $\tau_{12}$ and $\theta_{12}$ is not complete. To a certain extent he is right because instead of using eq 19 he had to calculate $\theta_{12}$ employing the three-state differential equations as presented in eqs 10a.

\section{Summary and Conclusions}

In this work, we studied the three ADT angles that form a 3 $\times 3$ ADT matrix by employing nonadiabatic coupling terms calculated from a diabatic potential energy matrix. To calculate these angles as a function of a cyclic variable (in this case an angle $\varphi$ which is defined in the range $[0,2 \pi]$ ), we employed a set of coupled first-order differential equations ${ }^{24}$ which are solved here for the first time. In fact, there are many ways to form the relevant system of equations for the ADT angles and each system may lead to a different set of angles. The solutions of the different sets of equations were found to be stable and did not cause any numerical difficulties. Their relevance was checked by comparing the ADT matrices as obtained by the different systems of equations. In all cases, the ADT matrices were found to be identical to each other.

A certain effort, in this study, was devoted to clarifying the relations between the various systems of equations. This effort ended with identifying two groups of systems of equations, where each system in a given group is identical to the other systems (in the same group) but contains different angles as the unknown functions. We also found that two systems of equations, each belonging to a different group (and therefore of a different form), will yield a similar solution (i.e., the same ADT angles) if solved for the appropriate boundary conditions. In this way, one (basic) group of systems of equations was identified which can be defined as the independent group and which contains three systems of equations, each with particular nonoverlapping features.

An interesting outcome is the fact that the ADT angle, $\theta_{12}$, calculated from eqs 10a overlaps very nicely, along most of the cycle expressed in terms of an angle $\varphi$, with the $\theta_{\mathrm{t} 12}$, the angle which follows from a direct integration over $\tau_{12}$ (see eq 19). This is in particular the case when the region surrounded by varying $\varphi$ contains only one isolated degeneracy. In this case, eq 19 is even capable of yielding the correct topological phase. If the region contains more than one point of degeneracy, the overlap between the two $\varphi$-dependent functions stops toward the end of the cycle and $\theta_{\mathrm{t} 12}$ misses the correct topological phase. In other words, if one is interested in the correct topological phases of a three-state system, he should consider solving the three-state equations and not rely on two-state equations.

A very important result of this study is that, consistently, all topological phases were either zero or $\pi$. This supports a recent theoretical study by the two present authors ${ }^{30}$ that says that an ADT matrix can yield diabatic states if and only if, upon a completion of a cycle, they are diagonal and have the numbers \pm 1 in their diagonal.

Acknowledgment. M.B. thanks Professors Y. T. Lee and S. H. Lin for their warm hospitality at the IAMS and the Academia Sinica for supporting this research. A.A. thanks Professor A. J. C. Varandas, for his warm hospitality at the University of Coimbra and for supporting this research.

\section{References and Notes}

(1) Baer, M.; Niedner-Shcattenburg, G.; Toennies, J. P. J. Chem. Phys. 1989, 91, 4169. Baer M.; Liao, C.-L.; Xu, R.; Flesch, G. D.; Nourbakhsh 
S.; Ng, C. Y. J. Chem. Phys. 1990, 93, 4845. Last, I.; Gilibert, M.; Baer M. J. Chem. Phys. 1997, 107, 1451.

(2) Aguilon, F.; Sizum, M.; Sidis, V.; Billing, G. D.; Markovic, N. J. Chem. Phys. 1996, 104, 4530. Aguilon, F.; Sizum, M.; Sidis, V.; Zenevich, V.; Billing, G. D.; Markovic, N. Chem. Phys. 1996, 209, 327. Grinberg, D.; Sidis, V.; Cobut, V. J. Chem. Phys. 1998, 108, 6331.

(3) Köppel, H.; Domcke, W.; Cederbaum, L. S. Adv. Chem. Phys. 1984, 57, 59; Pacher, T.; Cederbaum, L. S.; Köppel, H. Adv. Chem. Phys. 1993, $84,293$.

(4) Domcke, W.; Stock, G. Adv. Chem. Phys. 1997, 100, 1.

(5) Lepetit, B.; Kuppermann, A. Chem. Phys. Lett. 1990, 166, 581. Kuppermann, A.; Wu, Y.-S. M. Chem. Phys. Lett. 1993, 205, 577. Wu,

Y.-S. M.; Kuppermann, A. Chem. Phys. Lett. 1995, 235, 105.

(6) Markovic, N.; Billing, G. D. J. Chem. Phys. 1993, 99, 2674.

(7) Wu, X.; Wyatt R. E.; D'mello, M. J. Chem. Phys. 1994, 101, 2953.

(8) Sadigov, R. G.; Yarkony, D. R. J. Chem. Phys. 1998, 109, 20. Lengsfield, B. H.; Yarkony, D. R. In State Selected and State-to-Sate IonMolecule Reaction Dynamics: Part 2, Theory; Baer, M., Ng, C. Y., Eds.; John Wiley and Sons: New York, 1992; Vol. 82, p 1.

(9) Schon, J.; Koppel, H. J. Chem. Phys. 1995, 103, 9292.

(10) Sidis, V. In State Selected and State-to-Sate Ion-Molecule Reaction Dynamics: Part 2, Theory; Baer, M., Ng, C. Y., Eds.; John Wiley and Sons: New York, 1992; Vol. 82, p 73.

(11) Varandas, A. J. C.; Tennyson, J.; Murrel, J. M. Chem. Phys. Lett. 1979, 61, 431. Varandas, A. J. C.; Yu, H. G. Chem. Phys. Lett. 1996, 259, 336.

(12) Werner, H.-J.; Meyer, W. J. Chem. Phys. 1981, 74, 5802.

(13) Ruedenberg, K.; Atchity, G. J. J. Chem. Phys. 1993, 99, 3799.

(14) Dobbyn, A. J.; Knowels, P. J. Mol. Phys. 1997, 91, 1107.
(15) Alijah, A.; Nikitin, E. E. Mol. Phys. 1999, 96, 1399.

(16) Romero, T.; Aguilar, A.; Gadea, F. X. J. Chem. Phys. 1999, 110, 6219.

(17) Baer, R.; Charutz, D.; Kosloff, R.; Baer, M. J. Chem. Phys. 1996, 105, 9141. Charutz, D. M.; Baer, R.; Baer, M. Chem. Phys. Lett. 1997, $265,629$.

(18) Adhikari, S.; Billing, G. D. J. Chem. Phys. 1999, 111, 40

(19) Baer, M.; Englman, R. Chem. Phys. Lett. 1996, 265, 105.

(20) Baer, M. J. Chem. Phys. 1997, 107, 10662.

(21) Baer, M. J. Chem. Phys. 1998, 109, 891.

(22) Meiswinkel, R.; Köppel, H. Chem. Phys. 1990, 144, 117.

(23) Baer, M. Chem. Phys. Lett. 1975, 35, 112. Baer, M. Chem. Phys. 1976, 15,49 .

(24) Top, Z. H.; Baer, M. J. Chem. Phys. 1977, 66, 1363.

(25) Baer, M. In State Selected and State-to-Sate Ion-Molecule Reaction Dynamics: Part 2, Theory; Baer, M., Ng, C. Y., Eds.; John Wiley and Sons: New York, 1992; Vol. 82, p 187.

(26) Cocchini, F.; Upton, T. H.; Andreoni, W. J. Chem. Phys. 1988, 88,6068 .

(27) Baer, M.; Englman, R. Mol. Phys. 1992, 75, 293.

(28) Longuet-Higgins, H. C. Adv. Spectrosc. 1961, 2, 429.

(29) Zwanziger, J. W.; Grant, E. R. J. Chem. Phys. 1987, 87, 2954.

(30) Baer, M.; Alijah, A. Submitted.

(31) Yarkony, D. R. J. Chem. Phys. 1996, 105, 10456. Matsunaga, N.; Yarkony, D. R. J. Chem. Phys. 1997, 107, 7825. Yarkony, D. R. J. Chem Phys. 1999, 110, 701 .

(32) Chaban, G.; Gordon, M. S.; Yarkony, D. R. J. Phys. Chem. A 1997, 101,7953 . 